\title{
Des salariés associatifs moins bien payés ?
}

Une analyse sociohistorique et statistique par domaine d'activité What Pay Gap between Voluntary Associations and the Rest of the Private Sector? A Socio-Historical and Statistical Analysis According to Industries

\section{Laura Nirello et Lionel Prouteau}

\section{OpenEdition \\ Journals}

Édition électronique

URL : http://journals.openedition.org/travailemploi/7361

DOI : 10.4000/travailemploi.7361

ISSN : $1775-416 \mathrm{X}$

Éditeur

DARES - Ministère du Travail

\section{Édition imprimée}

Date de publication : 1 octobre 2016

Pagination : 31-54

ISSN : 0224-4365

\section{Référence électronique}

Laura Nirello et Lionel Prouteau, « Des salariés associatifs moins bien payés ? », Travail et Emploi [En ligne], 148 | octobre-décembre 2016, mis en ligne le 11 juillet 2019, consulté le 05 septembre 2019. URL : http://journals.openedition.org/travailemploi/7361 ; DOI : 10.4000/travailemploi.7361 


\title{
Des salariés associatifs moins bien payés ? \\ Une analyse sociohistorique et statistique par domaine d'activité
}

\author{
Laura Nirello ${ }^{* *}$, Lionel Prouteau ${ }^{* * *}$
}

\begin{abstract}
Les salariés du secteur associatif sont-ils moins payés que leurs homologues du reste du secteur privé ? Les recherches économiques comparatives menées à l'étranger ont révélé que la réponse à cette question varie selon les domaines d'activité. Le présent article se propose de contribuer à la compréhension de ces différences, qui sont peu documentées en France. À cette fin, il adopte une approche sociohistorique de la construction de la relation salariale dans les associations. Il s'interroge ainsi sur l'effet que peut exercer la présence de bénévoles sur les normes la structurant. Il examine également l'incidence que peuvent avoir sur elle les différences de temporalité des processus de professionnalisation et les degrés inégaux de développement des négociations collectives selon les domaines d'activité dans le monde associatif. À partir d'une exploitation des Déclarations annuelles des données sociales (DADS), l'article montre que dans le sport et le spectacle vivant, dont la professionnalisation est récente et qui sont d'importants utilisateurs de bénévolat, les salariés associatifs sont pénalisés par rapport au reste du privé, ce qui n'est le cas ni dans le domaine du social et du médico-social, ni dans celui de la santé.
\end{abstract}

$\mathrm{T}$ ravailler dans les associations est-il pénalisant en termes de rémunération? Dans le cadre des analyses économiques consacrées au secteur sans but lucratif, la question a donné lieu à différentes hypothèses. Bien que non exclusive, celle qui domine ces recherches est l'hypothèse du don de travail, qui postule que les salariés associatifs

\footnotetext{
* Ce travail a bénéficié d'une aide de l'État gérée par l'Agence nationale de la recherche au titre du programme Investissements d'avenir portant la référence ANR-10-EQPX-17 (Centre d'accès sécurisé aux données - CASD). Les auteurs remercient trois rapporteurs anonymes pour leurs remarques et suggestions. Ils restent évidemment seuls responsables des éventuelles erreurs qui demeureraient dans cet article.

** Laboratoire d'économie et de management de Nantes-Atlantique (Lemna), université de Nantes ; laura.nirello@univ-nantes.fr.

*** Lemna, université de Nantes ; lionel.prouteau@ univ-nantes.fr.
} 
acceptent des rémunérations inférieures à celles auxquelles ils pourraient prétendre ailleurs, en contrepartie de gratifications intrinsèques induites par leur activité professionnelle, liées notamment aux bénéfices sociaux qu'elle engendre.

Les travaux empiriques réalisés sur le sujet, aux États-Unis en particulier, montrent toutefois que la comparaison entre salaires du secteur sans but lucratif et ceux du secteur lucratif conduit à des constats variables selon les domaines d'activité. Une telle disparité des situations est également observée en France, mais reste assez peu documentée. Pour contribuer à son étude, le présent article porte un regard historique sur la relation salariale, qui est appréhendée sous l'angle d'une construction sociale dans des contextes associatifs singuliers, puisqu'ils impliquent des acteurs spécifiques que sont les bénévoles. Nous y mettons l'accent sur les différences chronologiques dans les processus de professionnalisation et d'élaboration des normes collectives régissant la détermination des salaires dans les associations. Nous faisons l'hypothèse que c'est dans les domaines où ces processus sont les plus récents et l'usage du bénévolat le plus intense que les rémunérations au sein du secteur associatif sont inférieures à celles du secteur privé non associatif.

La comparaison entre les deux secteurs ne se limite pas ici au seul salaire horaire, comme tel est généralement le cas dans la littérature sur le sujet, mais elle est élargie au salaire annuel des postes occupés par les salariés. Si l'examen du premier est indispensable, il ne saurait suffire. En effet, les écarts de revenus sont également déterminés par des différences dans les volumes horaires des postes de travail. Dans un autre contexte de recherche, Dominique Meurs et Sophie Ponthieux (2006) soulignent que ces différences expliquent une partie substantielle des écarts salariaux entre les hommes et les femmes.

L'article commence par un rapide survol de la littérature économique traitant de la comparaison entre les salaires des secteurs lucratif et sans but lucratif. Il aborde ensuite la dimension sociohistorique de la constitution de la relation salariale en milieu associatif et souligne la diversité des scénarios rencontrés dans plusieurs domaines d'activité, ce qui conduit à spécifier notre hypothèse quant à l'écart prédictible de salaire entre les associations et le reste du secteur privé dans ces domaines ${ }^{1}$. Nous présentons ensuite les données utilisées pour notre étude empirique avant d'exposer et de commenter les résultats de notre investigation.

1. Par commodité rédactionnelle, les employeurs privés non associatifs sont généralement désignés ici par les expressions « reste du secteur privé » ou « reste du privé ». 


\section{Les salaires associatifs dans l'analyse économique : don de travail versus salaire d'efficience}

Pourquoi les salaires dans les associations différeraient-ils de ceux en vigueur dans le reste du secteur privé ? Les développements consacrés par la littérature économique à cette question ne conduisent pas à des prédictions univoques. L'hypothèse la plus connue est celle du don de travail (WeIsBrod, 1983 ; PRESTON, 1989). Elle prête aux salariés des organisations sans but lucratif (OSBL) un souci pour l'intérêt général et pour les externalités sociales positives engendrées par les services rendus au public. Elle leur attribue de ce fait une disposition spécifique à accepter des salaires moindres en contrepartie des gratifications intrinsèques procurées par ces externalités ${ }^{2}$. Il s'agit là d'une approche des rémunérations qui renvoie à une analyse en termes de différences compensatoires (ROSEN, 1986). Les stratégies de recrutement des employeurs associatifs peuvent alors jouer de cette disposition en encourageant un processus d'autosélection des salariés à l'embauche. L'argument a été développé par Femida HANDY et Eliakim KATZ (1998) à partir de l'analyse des OSBL que fait Henry B. HANSMANN (1980), pour qui ces organisations existent du fait de leur aptitude à engendrer la confiance des consommateurs sur des marchés à forte asymétrie d'information. De telles situations sont en effet susceptibles d'entraîner des comportements opportunistes de la part des producteurs et la qualité des services peut en être affectée. Les OSBL inspireraient davantage confiance que les entreprises lucratives car, du fait de leur soumission à la contrainte légale et fiscale de non-distribution du profit, elles seraient censées ne pas avoir d'incitations à tirer avantage de cette asymétrie. F. HANDY et E. KATZ (1998) soulignent que ce problème de confiance entre producteurs et consommateurs se double d'un problème de confiance interne à l'organisation (du type principal-agent) dès lors que les responsables bénévoles des OSBL qui siègent dans leurs organes dirigeants ne disposent pas des indicateurs traditionnels associés à la recherche du profit pour mesurer l'efficacité des salariés, notamment des cadres. Pour attirer un personnel dévoué et attaché à la qualité des prestations plus qu'aux rétributions purement monétaires, l'employeur non lucratif proposera des salaires inférieurs à ceux en vigueur dans le secteur lucratif tout en veillant à la satisfaction des motivations intrinsèques des salariés.

D'autres approches théoriques amènent à des conjectures différentes. Les analyses en termes de droit de propriété prédisent des salaires associatifs supérieurs à ceux du reste du secteur privé au motif qu'en l'absence, pour ces associations, de droit d'appropriation du surplus résiduel (c'est-à-dire puisqu'il leur est impossible de distribuer le profit net après le paiement de toutes les charges), il n'y a pas d'incitation

2. Les gratifications intrinsèques sont celles qui sont liées à la pratique de l'activité en tant que telle. Elles peuvent résider entre autres choses dans l'intérêt des tâches ou le sentiment du salarié de concourir à un travail d'utilité sociale. Les gratifications extrinsèques sont externes à l'activité proprement dite, elles en sont séparables. Elles peuvent être procurées par exemple par la rémunération, les perspectives de promotion offertes, etc. 
à minimiser les coûts (PRESTON, 1988). Dans un esprit assez proche, l'hypothèse d'une moindre efficience dans les OSBL du fait d'une plus grande discrétion managériale ${ }^{3}$ (HANSMANN, 1980) se traduirait par une vigilance moindre à l'égard des coûts salariaux et donc des rémunérations plus élevées. Une analyse en termes de salaires d'efficience peut également conduire à postuler des rémunérations supérieures dans les OSBL. Afficher le souci de la qualité des services rendus incite en effet ces organisations à recruter des salariés partageant cette préoccupation. Puisque la qualité du travail ne peut être déterminée ex ante (sélection adverse) et parce qu'il a besoin de s'assurer de l'implication future des salariés dans leurs missions (aléa moral), l'employeur non lucratif est susceptible d'offrir des salaires supérieurs à ceux du secteur lucratif (LEETE, 2006). Parce qu'elle ignore l'hypothèse de satisfactions intrinsèques du travail dans les OSBL, cette approche en termes de salaires d'efficience conduit à des conclusions opposées à celles de F. HANDY et E. KaTZ (1998).

Les prédictions tirées de la littérature économique sur la comparaison des salaires entre les deux secteurs s'avèrent donc ambiguës. Les travaux empiriques menés sur le sujet, particulièrement aux États-Unis, conduisent à des résultats qui ne sont pas aisément comparables entre eux dans la mesure où les méthodes d'investigation et les périmètres d'observation peuvent varier ${ }^{4}$. Néanmoins, lorsque les caractéristiques des emplois occupés et les domaines d'activité sont contrôlés dans les équations de salaires, le différentiel de rémunération, qui apparaissait défavorable dans un premier temps aux OSBL, se réduit, voire devient nul (LEETE, 2001 ; RUHM, BORKOSKI, 2003), même si Anne E. PRESTON et Daniel W. SACKS (2010) soulignent qu'il reste non négligeable pour les fonctions managériales. Cette incidence de la prise en compte des domaines d'activité sur l'estimation de l'écart salarial entre les secteurs sans but lucratif et lucratif traduit la concentration de l'emploi du premier dans des domaines d'activité où les rémunérations sont plutôt faibles (social et médico-social en particulier). Pour autant, cela ne signifie pas que les salaires des deux secteurs sont les mêmes dans chaque domaine où ils coexistent. A. E. PRESTON et D. W. SACKS (2010) montrent que les situations diffèrent d'un domaine à l'autre, les salariés des OSBL étant tantôt mieux, tantôt moins bien rémunérés que leurs homologues du secteur lucratif. Cette disparité des situations nous paraît être un sujet important de réflexion et de recherche.

Concernant la France, Mathieu NARCY (2009) trouve que les salariés associatifs travaillant à temps plein et occupant des emplois non précaires acceptent des rémunérations horaires qui sont de $12 \%$ inférieures en moyenne à celles qu'ils auraient pu percevoir dans le secteur privé hors associations. Il en conclut que la thèse du don de travail est valide. Matthieu HÉly (2008, p. 128) soutient également que l'une des «particularités les plus frappantes» du secteur associatif réside dans le fait qu' «à emploi égal, le salaire perçu diffère sensiblement de ce qu'il pourrait être dans le

3. La discrétion managériale est la marge d'autonomie dont disposent les managers par rapport aux actionnaires dans une société de capitaux ou par rapport au conseil d'administration bénévole dans une association.

4. Pour une présentation succincte de certaines de ces études empiriques voir RUHM, BoRKOSKI (2003), LEETE (2006) ainsi que Preston, SACKS (2010). 
cadre d'une entreprise privée à but lucratif ». Mais il y voit l'effet d'une contrainte engendrée par l'absence d'alternatives sur le marché du travail plutôt que le fruit d'un choix délibéré de la part des salariés, même si ces derniers peuvent être amenés à faire « de nécessité vertu » (HÉLY, 2009).

$\mathrm{Si}$, dans l'équation de salaires qu'il estime, M. NARCY (2009) intègre bien les domaines d'activité, en revanche il ne mène pas l'examen comparatif entre les deux secteurs au niveau de chacun de ces domaines. Mobilisant les Déclarations annuelles de données sociales (DADS), Laurent BISAULT (2012) souligne que si les salaires moyens dans le monde associatif sont inférieurs à ceux du privé non associatif, ils sont sensiblement les mêmes dans certains types d'activité et même légèrement supérieurs dans les domaines de la santé et de l'hébergement médico-social. Mais les comparaisons effectuées concernent les salaires estimés en équivalent temps plein ; or l'auteur insiste sur le fait qu'il importe de tenir compte des différences de temps de travail, ce qui n'est que très rarement le cas des études évoquées plus haut qui considèrent le seul salaire horaire ${ }^{5}$.

De cette rapide incursion dans la littérature économique traitant des salaires associatifs, un enseignement important nous semble se dégager en ce qui concerne le niveau pertinent auquel la comparaison avec le secteur privé hors associations gagne à être menée : réalisé à l'échelle de l'ensemble de l'économie, l'exercice risque fort de conduire à des généralisations hâtives masquant la diversité des situations par domaine d'activité. C'est donc à un niveau désagrégé de la nomenclature des activités qu'il nous parait judicieux de conduire l'investigation, tout en veillant évidemment à ce que les deux secteurs qui font l'objet de la comparaison soient présents au niveau choisi.

Les analyses économiques que nous avons évoquées appréhendent la relation entre employeurs et salariés essentiellement au travers du prisme de l'échange marchand, même si elles prennent en compte certaines particularités de la transaction, telles que les asymétries d'information et la nature particulière de la fonction-objectif des OSBL. Or la relation salariale excède le cadre de l'échange marchand. Elle n'est pas d'ordre ponctuel et ne peut être réduite à la signature du contrat de travail. Elle se présente comme un « processus continuel de conflits et de négociations » (BAZzoli et al., 1994, p. 98). Elle est organisée par des normes, explicites ou implicites, régissant l'entreprise et le domaine d'activité. Elle est soumise à des accords collectifs d'entreprise, de branche ou interprofessionnels. Comparer les salaires dans les associations à ceux du reste du secteur privé requiert donc un détour sociohistorique permettant d'éclairer les particularités du monde associatif au regard de ces aspects organisationnels et institutionnels pour comprendre en quoi ces particularités sont susceptibles d'influencer la construction des relations salariales dans les associations. Telle est l'approche que nous privilégierons dans cet article.

5. L'une des rares exceptions est l'étude de Christopher J. RuHM et Carey BorKosKi (2003) qui porte sur le salaire hebdomadaire. 


\section{Contextualiser et historiciser l'étude des relations salariales dans les associations}

Le développement du rôle économique des associations a été spectaculaire depuis le milieu du $\mathrm{XX}^{\mathrm{e}}$ siècle et s'est accompagné d'une croissance rapide et importante du salariat, tendanciellement plus forte que celle de l'emploi de l'ensemble du secteur privé. Le secteur associatif accueille ainsi plus d'un million huit cent mille salariés en 2014, ce qui représente 7,5\% de l'emploi total français en équivalent temps plein ${ }^{6}$. Cet essor du salariat a pour particularité d'intervenir dans des organisations mobilisant également un autre type de ressources humaines, à savoir le bénévolat. Or une telle cohabitation est considérée par le CONSEIL D’ÉTAT (2000, p. 277) comme « une source de confusion ou de difficultés concernant le statut social des personnes travaillant pour la même association » et par M. HÉLY (2009) comme un facteur de fragilisation de la légitimité des salariés. En quoi peut-elle affecter la détermination des salaires ?

Un premier élément de réponse réside dans la difficulté qu'ont parfois certains bénévoles administrateurs à se reconnaître comme employeurs et à en assumer les responsabilités en matière de relations professionnelles et de dialogue social (HÉLY et al., 2015). Dans ce cas de figure, les salariés comme leurs rémunérations ont à en subir les conséquences. Par ailleurs, la présence de bénévoles, notamment lorsqu'ils ont à accomplir des tâches opérationnelles et sont mobilisés dans le cours même de l'activité de production aux côtés de la main-d'œuvre rémunérée ${ }^{7}$, favorise l'alimentation d'un ethos de l'engagement qui peut avoir des «effets de débordement» sur les salariés, dans la mesure où il instille dans le registre du travail subordonné une logique d'engagement désintéressé conduisant à relativiser l'importance accordée à la rétribution monétaire. Une telle situation n'est pas sans lien avec la théorie du don de travail dont il a été question ci-dessus, même si cette inclination pour le don ne relève pas nécessairement d'un trait de personnalité des salariés ou d'une motivation qui préexisteraient à la relation salariale. Elle peut tout aussi bien être induite par la spécificité de cette relation dès lors que la propension au don est une condition de la reconnaissance des salariés par les bénévoles et l'association employeuse. Enfin, dans un autre ordre d'idées, la présence de bénévoles opérationnels aux côtés des salariés peut révéler la fragilité économique de l'association, qui se traduit concrètement par des emplois précaires et des rémunérations faibles.

Ainsi, la coexistence du salariat et du bénévolat, auxquels s'ajoute le cas échéant le volontariat ${ }^{8}$, offre un contexte propice à un mélange des genres ne facilitant pas la reconnaissance d'une relation salariale de droit commun. Selon l'enquête du Centre

6. Voir le site de l'Insee : https://www.insee.fr/fr/statistiques/2505833?sommaire=2506122, consulté le 17 mai 2017.

7. Les bénévoles opérationnels se distinguent de ceux siégeant dans les instances dirigeantes (conseils d'administration, bureaux).

8. Le volontaire (notamment dans le cadre du service civique) a un statut donnant droit à une indemnité, ce qui n'est pas le cas du bénévole. Contrairement à celui du salarié, son statut ne relève pas du Code du travail. 
d'économie de la Sorbonne - CNRS conduite en 2011-2012 (TCHERNONOG, 2013), l'ampleur de cette coexistence est variable selon le domaine d'activité : ainsi, le sport, la culture et les loisirs mobilisent plus de $45 \%$ des heures de bénévolat des associations employeuses et n'occupent que $13 \%$ du volume horaire de l'emploi associatif ; les équilibres sont bien différents dans le domaine sanitaire et social, qui représente plus de $60 \%$ du volume de l'emploi salarié mais moins de $40 \%$ du bénévolat des associations employeuses, en particulier dans l'action sociale sans hébergement (activités caritatives, aide à domicile, etc. $)^{9}$.

Dans les associations culturelles, sportives et de loisirs, la porosité des frontières entre condition bénévole et statut salarié est confirmée par divers travaux sociologiques. À propos du secteur sportif, Caroline Снімот et Manuel SсноттÉ (2006) notent qu'au nom de la " passion sportive », les salariés sont conduits à articuler registre de la vocation et régime du travail. Dans un même ordre d'idées, Marc FALCOZ et Emmanuelle WALTER (2007, p. 82) écrivent que travailler dans un monde de bénévoles incite les salariés à incorporer « la logique du don comme une condition sine qua non de leur intégration dans l'espace club». Dans le secteur culturel et particulièrement dans le domaine du spectacle vivant, les associations recourent massivement au bénévolat (DEROIN, 2014). Étudiant le cas des musiques actuelles, Carole LE RENDU-LizÉE (2005, p. 26) souligne ainsi que « la reconnaissance n'est pas liée à des éléments statutaires ou de rémunération mais essentiellement à une forme d'attachement affectif, moral et donc intrinsèque à l'activité ». Dans les loisirs, Francis Lebon (2009, p. 5 et 6) constate que « l'animation permet la transformation du militantisme en engagement professionnel » et que « les centres de loisirs représentent un marché du travail ouvert où les logiques du "don" et de la "passion" sont très présentes ». Les conditions d'accès au métier d'animateur participent de cette confusion des frontières entre bénévolat et salariat dès lors que les « clefs d'accès usuelles à l'espace professionnel de l'animation » sont des diplômes non professionnels, à savoir le brevet d'aptitude aux fonctions d'animateur (BAFA) et le brevet d'aptitude aux fonctions de directeur (BAFD) (LEBON, 2009, p. 27). On observe dans ces différents champs ce que Maud SimONET (2006, p. 204) appelle « la circulation et la démultiplication des individus dans la plupart des statuts offerts ». Il n'est pas rare, en effet, que les salariés des associations y soient d'anciens bénévoles.

La coprésence du bénévolat et de l'emploi rémunéré a également caractérisé la phase d'émergence du salariat associatif dans les activités de service social de la première moitié du $\mathrm{Xx}^{\mathrm{e}}$ siècle et a influé sur les qualités attendues de ceux que l'on appellera plus tard les travailleurs sociaux. Christine MiAS (1998, p. 28) rappelle ainsi que les assistantes sociales ont été initialement recrutées «sur un mode de sélection valorisant l'idée de vocation, de charisme, de valeurs morales ». Mais cette phase originelle a cédé progressivement la place à un processus d'affirmation des identités professionnelles qui ont affirmé leur légitimité en opposition au bénévolat ${ }^{10}$.

9. Dans l'hébergement social et médico-social, le bénévolat opérationnel est nettement moins présent.

10. Jacques Ion (2005, p. 150) écrit à ce propos que « le travail social [...] s'est constitué contre le bénévolat ». 
La salarisation qui a accompagné la professionnalisation des champs d'action du monde associatif s'est mise en place selon des chronologies différentes en fonction des domaines d'activité ${ }^{11}$. Dans la santé, le social et le médico-social, c'est au cours des années 1950 à 1980 que les effectifs salariés augmentent. Dans l'animation mais aussi dans le sport, le développement de l'emploi est postérieur et intervient surtout dans les années 1980 et 1990. L'emploi culturel, quant à lui, s'est très fortement accru aux cours des dernières décennies : les effectifs des professions des spectacles augmentent notamment de $95 \%$ entre 1991 et 2011 (GOUYON, PATUREAU, 2014) en raison de l'émergence de champs comme celui des musiques actuelles. Or, nous l'avons déjà mentionné, c'est précisément dans le spectacle vivant que la présence associative est la plus forte dans le monde culturel ${ }^{12}$.

Les différences de chronologies des processus de professionnalisation selon les domaines d'activité expliquent en partie l'hétérogénéité actuelle du recours au bénévolat au sein du monde associatif. Cet asynchronisme éclaire également leurs degrés inégaux de développement des relations professionnelles et du dialogue social et se manifeste par la diversité des normes qui, dans les associations, structurent la relation salariale. Ainsi, en matière de négociations de branche dans le secteur associatif, c'est dans les domaines de la santé, du social et du médico-social que l'histoire est la plus ancienne. La convention collective des établissements privés d'hospitalisation, de soins, de cure et de garde à but non lucratif est signée en octobre 1951. Elle concerne les personnels des établissements d'hébergement des personnes âgées dépendantes (Ehpad) ainsi que ceux des établissements sanitaires. En mars 1966, la convention collective de travail des établissements et services pour personnes inadaptées et handicapées est à son tour ratifiée, couvrant les établissements pour l'enfance protégée, handicapée, inadaptée, et ceux pour adultes handicapés, etc. Dans le domaine de l'aide à domicile, la convention collective nationale des personnels des organismes de travailleuses familiales date de 1970, de même que la convention collective nationale des aides familiales rurales et du personnel de l'aide à domicile en milieu rural (ADMR) ; les deux conventions fusionnent en 2010 avec celle des associations d'aide à domicile de 1983, pour constituer la convention de branche de l'aide à domicile. Ces accords collectifs ont été favorisés par une structuration plus précoce, et qui reste encore aujourd'hui plus affirmée, des associations employeuses dans le champ de l'aide aux personnes (RAVON, Ion, 2012). Ainsi la Fédération des établissements hospitaliers et d'aide à la personne (Fehap) est créée en 1936, l'Union nationale des associations de parents, de personnes handicapées mentales et de leurs amis (Unapei) en 1960, le Syndicat national des associations pour la sauvegarde de l'enfant à l'adulte (Snasea)

\footnotetext{
11. La professionnalisation concerne tant les activités que les personnes qui les exercent et les organisations dans lesquelles elles s'insèrent. La professionnalisation des activités s'entend ici selon la définition qu'en donne Raymond BouRdonCle (2000), c'est-à-dire comme le passage de ces dernières au statut de métiers et de professions. La professionnalisation des individus se comprend comme l'acquisition de savoirs et de compétences qui les rendent aptes à exercer ces métiers. La professionnalisation de l'organisation est définie comme un processus de rationalisation de l'action collective en vue de la réalisation des activités (CHANTELAT, 2001).

12. En 2006, les associations représentent $77 \%$ des organismes du spectacle vivant (URRUTIAGUER, 2009).
} 
en 1961 et le Syndicat général des organismes privés sanitaires et sociaux à but non lucratif (SOP) en $1962^{13}$.

Un certain nombre d'activités de loisirs sont régies par la convention collective de l'animation dont le champ d'application est toutefois plus vaste. Elle date de 1988 et est donc postérieure aux principales conventions du domaine social. D'après F. LEBON (2009, p. 105), sa signature « ne semble pas avoir eu d'incidence majeure sur les statuts d'emploi ». Cet auteur souligne ainsi la faible mobilisation du groupe professionnel des animateurs, l'absence d'une association nationale les regroupant et la grande faiblesse de l'implantation syndicale, autant d'éléments qui suggèrent un rapport de force défavorable aux salariés.

Le sport est le domaine dans lequel le vide conventionnel a prévalu le plus longtemps, puisqu'il a fallu attendre 2005 pour que soit signée une convention collective après que les employeurs ont enfin réussi à se fédérer et à assurer leur représentation dans le dialogue social de branche par la constitution du Conseil social du mouvement sportif (CoSMos) en avril 1997 (FLEURIEL, 2013).

S'agissant enfin de la culture, et plus particulièrement du spectacle vivant, la situation est assez spécifique. Qu'il soit ou non associatif, le secteur privé y dispose de trois conventions jusqu'en 2013, date à laquelle elles fusionnent : la convention collective nationale étendue des théâtres privés (1977), la convention collective étendue régissant les rapports entre les entrepreneurs de spectacles et les artistes dramatiques, lyriques, marionnettistes, de variété et musiciens en tournée (2003) et la convention collective (non étendue) chanson, variétés, jazz, musiques actuelles (2003). Or ces conventions collectives sont mal connues et peu respectées (CORSANI, LAZZARATO, 2008), notamment parce que le régime de l'intermittence permet aux structures d'externaliser une partie du coût salarial vers l'assurance chômage, et est par conséquent de fait investi d'un « rôle beaucoup plus central que la plupart des autres textes conventionnels » (Proust, 2010, p. 379). Il en résulte ce que Pierre-Michel Menger (2010) appelle un défaut d'engagement de la responsabilité d'employeur et un système désintégré d'emploi se traduisant par un « délitement de la norme salariale » (PROUST, 2012, p. 67). Dans les associations, un tel délitement est susceptible d'être aggravé par la fragilité de leurs modèles économiques et leur dépendance aux financements publics (Bureau, SCHMidT, 2004). C'est donc plus particulièrement dans les organismes à statut associatif que le travail gratuit est une condition de viabilité de la structure (Proust, 2010).

Dans ce qui précède, nous avons mis l'accent sur les rythmes différenciés de développement du salariat et de construction des relations professionnelles dans les associations, ainsi que sur les formes idiosyncrasiques des processus de professionnalisation. Dans le domaine social et médico-social, et plus spécifiquement dans les structures d'hébergement, ce processus a eu pour corollaire la relégation des bénévoles

13. Snasea et SOP fusionnent en 2010 pour constituer le Syndicat des employeurs associatifs de l'action sociale et médico-sociale (Syneas). 
dans des fonctions d'administrateurs, si bien que la cohabitation des bénévoles et des salariés dans la réalisation des activités de service y est devenue rare. Dans le sport, la culture et les loisirs, même si elle s'est accompagnée d'une croissance du travail salarié par rapport au travail non rémunéré, la professionnalisation n'a pas eu le même effet d'éviction du bénévolat dont l'importance symbolique de l'engagement continue à conférer une forte dimension vocationnelle aux métiers qui y sont exercés. Nous pouvons donc avancer l'hypothèse que dans ces domaines, la comparaison entre les salaires associatifs et ceux du reste du privé sera plus défavorable aux premiers tandis que dans la santé, le social et le médico-social, nous n'avons pas lieu de conjecturer une différence notable de rémunérations entre les deux secteurs. Il est même possible que dans certains champs, comme celui de l'aide à domicile où la pénétration du secteur privé lucratif est récente puisqu'elle n'intervient qu'à la suite de la loi Borloo de 2005, le salaire associatif soit plus élevé. Avant de tester empiriquement de telles hypothèses, notons qu'elles convergent au moins partiellement avec celles qui pourraient être tirées des approches économiques présentées dans notre première partie. En effet, la forte présence bénévole dans une association atteste peut-être l'existence de gratifications intrinsèques susceptibles d'encourager les comportements de don de travail des salariés. Dans le même temps, elle permet un contrôle plus étroit de la main-d'œuvre rémunérée, minimisant le risque d'aléa moral et affaiblissant ainsi la pertinence de l'hypothèse de salaires d'efficience (HAIDER, SCHNEIDER, 2010).

\section{Données utilisées, secteurs et domaines d'activités retenus}

Pour notre investigation empirique, nous utilisons les Déclarations annuelles de données sociales (DADS) de l'année 2010. Les DADS sont des fichiers administratifs, communs aux administrations fiscales et sociales, que les employeurs doivent compléter annuellement. Elles couvrent l'ensemble des employeurs et de leurs salariés hormis les agents des organismes de l'État, les salariés des services domestiques et des activités extraterritoriales. Les fichiers de diffusion, appelés «DADS - Grand format », sont cependant complétés à partir d'autres sources et assurent ainsi une couverture exhaustive du salariat. Les chercheurs ont la possibilité de les exploiter à l'exception de ceux qui sont collectés par la Direction générale des finances publiques (DGFiP). Cette amputation de la base de données affecte surtout certaines activités relatives à l'agriculture. Bien que regrettable, elle a un effet très limité pour le présent article.

L'unité d'observation des $D A D S$ est le poste. Il correspond à la présence d'un salarié dans un établissement, quel que soit le nombre de périodes d'emploi qu'il a connues en cours d'année au sein de cet établissement. Si un salarié a travaillé au cours de l'année considérée dans deux établissements différents, il apparaît alors dans les $D A D S$ sur deux postes distincts. Les $D A D S$ fournissent des informations sur la nature des postes occupés, le nombre d'heures effectuées et les rémunérations versées (brutes et nettes). Nous utilisons dans cet article les salaires nets, autrement dit les salaires 
obtenus après déduction des cotisations de sécurité sociale, des régimes de retraite et de prévoyance complémentaire, d'assurance chômage, et après décompte des contributions sociales généralisées (CSG) et des contributions au remboursement de la dette sociale (CRDS), déductibles et non déductibles. Les salaires nets comprennent la partie de l'intéressement non placée sur un plan d'épargne salariale. Les DADS permettent également de connaître certaines caractéristiques des entreprises et établissements employeurs. L'âge et le sexe des salariés sont renseignés mais leur niveau de diplôme et leur ancienneté dans l'établissement ne le sont pas.

Dans ce travail, nous avons fait le choix de ne retenir que le «noyau dur» de l'emploi. Nous ne prenons pas en compte les postes annexes (encadré 1), suivant en cela l'Institut national de la statistique et des études économiques (Insee), qui les exclut de ses statistiques sur l'emploi et les salaires car ils ne sont pas considérés comme de « vrais emplois ». Ont également été écartés de l'analyse les contrats d'intérimaires (qui sont déclarés par les agences d'intérim et ne peuvent être rattachés aux entreprises dans lesquelles ils travaillent), les contrats d'apprentissage, les postes sans contrat qui recouvrent des réalités composites et les emplois aidés ${ }^{14}$. Pour l'investigation au niveau agrégé des activités, il nous a semblé plus judicieux de limiter le périmètre de l'étude aux services puisqu'ils constituent le domaine quasi exclusif de l'activité des associations.

Nous écartons également les postes du secteur public en raison des modalités particulières d'accès aux emplois de fonctionnaires (par concours) et des normes qui déterminent la progression de leurs salaires au cours de la vie professionnelle. Certes, la fixation des rémunérations dans le secteur privé est également encadrée par la loi ou par des conventions collectives, mais les employeurs n'en conservent pas moins des marges de manœuvre qui donnent tout son sens à la comparaison envisagée.

\section{ENCADRÉ 1}

\section{Postes non annexes, postes annexes}

L'Insee distingue deux types de postes : les postes non annexes et les postes annexes. La distinction est établie sur la base d'un seuil conventionnel, que l'on peut définir comme suit. Pour être considéré comme non annexe, un poste doit avoir une rémunération annuelle supérieure à trois Smic (salaire minimum interprofessionnel de croissance) mensuels ou bien compter un volume annuel d'heures supérieur à 120, une durée annuelle de l'emploi supérieure à 30 jours et avoir un rapport volume horaire/durée supérieur à 1,5. Si aucune

14. Nous avons exclu les emplois aidés de notre investigation en raison de leur caractère transitoire. Certes, une fois le contrat aidé terminé, il n'est pas impossible que le salarié reste dans l'association mais telle n'est pas la règle. Ainsi, en 2012, six mois après leur sortie du contrat, $20 \%$ des salariés ayant occupé un contrat d'accompagnement dans l'emploi (CUI-CAE) en association hors ateliers et chantiers d'insertion (ACI) étaient employés dans la même association, $20 \%$ l'étaient chez un autre employeur et $60 \%$ étaient sans emploi ou en emploi non salarié. Lorsque le contrat aidé s'était déroulé en ACI, ces pourcentages étaient respectivement de $4 \%, 17 \%$ et $80 \%$ (DURAND, 2014). Même s'il n'est pas systématique, le caractère transitoire du contrat aidé correspond bien à la grande majorité des cas. 
de ces deux conditions (en termes de rémunération ou de durée) n'est remplie, le poste est considéré comme annexe. Les postes non annexes constituent donc de « vrais » emplois, tandis que les postes annexes renvoient à des emplois occasionnels. Si un même individu occupe plusieurs postes non annexes au cours de l'année, l'Insee identifie un poste principal qui correspond à celui procurant le salaire net le plus élevé.

Le choix, qui suit la pratique de l'Insee, de ne retenir que les postes non annexes a toutefois un effet différent selon que l'on s'intéresse aux secteurs associatif ou privé hors associations. En effet la fréquence des postes annexes est plus élevée dans les associations puisqu'ils y représentent le tiers de l'ensemble des postes, contre 22,7\% dans le secteur privé non associatif (27,6\% si l'on se limite aux seules activités tertiaires).

Les domaines d'activité que nous avons choisi d'étudier sont ceux dont il a été question dans notre partie précédente : social et médico-social, santé, spectacle vivant, sports et loisirs (encadré 2). Sont ainsi concernés par notre étude $59 \%$ de l'ensemble des postes associatifs, contre un peu moins de $8 \%$ des postes du reste du secteur privé.

\section{ENCADRÉ 2}

\section{Périmètre des secteurs et domaines d'activité étudiés}

Nous avons identifié comme associations tous les organismes dont le code de la nomenclature des catégories juridiques de niveau II est « 92 », en excluant les congrégations. Le reste du secteur privé est donc constitué de toutes les autres structures de codes différents relevant du droit privé.

Le domaine du social et du médico-social comprend les activités couvertes par les codes 87 et 88 de la Nomenclature d'activités française révision 2 (NAF rév.2) de niveau 2. Nous étudions plus particulièrement deux sous-champs : celui des établissements d'hébergement pour personnes âgées dépendantes (Ehpad) et celui de l'aide à domicile, dans lesquels coexistent structures associatives et autres organisations de droit privé. Comme nous l'avons déjà mentionné, le bénévolat opérationnel y a quasiment disparu, ce qui n'est pas le cas dans les associations caritatives, humanitaires et plus généralement dans celles de l'action sociale sans hébergement pour lesquelles la comparaison avec le reste du secteur privé n'est pas envisageable puisque ce dernier en est absent. Les Ehpad sont regroupés sous le code $8710 \mathrm{~A}$ du niveau 5 de la NAF et l'aide à domicile sous le code $8810 \mathrm{~A}$. La santé correspond au code 86 du niveau 2 de la NAF. S' agissant du sport et des loisirs, nous avons conduit l'examen à deux niveaux. Le premier est celui du code 93 du niveau 2 de la NAF qui concerne l'ensemble des activités sportives, récréatives et de loisirs. Le second niveau s'intéresse à deux sous-champs particuliers de cet ensemble : les activités de clubs de sports (code 9312Z) et les autres activités récréatives et de loisirs (code 9329Z; par exemple, parcs de loisirs, stations de ski). Nous avons choisi ces deux sous-champs car la présence associative y est forte, puisqu'ils comprennent $89 \%$ des postes des associations relevant du code 93. Enfin, pour ce qui est de la culture, nous avons retenu les arts du spectacle vivant (code 9001Z), qui regroupent à eux seuls $58 \%$ des postes associatifs du domaine culturel. 


\section{Des salaires qui ne sont pas nécessairement inférieurs dans le secteur associatif}

Nous procédons en deux temps. Tout d'abord, par une analyse de statistiques descriptives, nous étudions les salaires annuels perçus, ce qui nous conduit à prendre en compte le temps de travail afférent à ces postes. Puis, nous nous penchons sur le salaire horaire en mobilisant des méthodes économétriques.

\section{Analyse descriptive des salaires annuels}

La comparaison entre les salaires annuels associatifs et ceux du reste du privé débouche sur des constats sensiblement identiques selon qu'elle est réalisée à partir des valeurs moyennes ou des valeurs médianes. Pour alléger le propos, la présentation des résultats ne retient que ces dernières, les tableaux permettant de prendre connaissance également des moyennes. Dans l'ensemble des services, le salaire net annuel médian d'un poste associatif est de plus de $16 \%$ inférieur à celui d'un poste dans le reste du secteur privé, toutes catégories socioprofessionnelles (CS) confondues (tableau 1). Cette différence a pour corollaire un écart de même nature au niveau des temps de travail, traduisant la plus forte fréquence des temps partiels en milieu associatif (seulement $56 \%$ des postes y sont à temps plein, contre $75 \%$ dans le reste du privé). Néanmoins l'écart observé entre les salaires annuels est assez nettement supérieur à celui des temps de travail, ce qui suggère que les rémunérations horaires sont également plus faibles dans les associations.

Obtenus à un niveau très agrégé, ces premiers résultats appellent un examen complémentaire sur au moins deux aspects : celui de la CS et celui des domaines d'activité. Le tableau 1 montre ainsi que toutes les CS sont affectées par le fait que les salaires dans le milieu associatif sont plus bas que dans le secteur privé non associatif. Toutes ont également des temps de travail plus faibles que dans le privé, à l'exception des employés qui, eux, ont des volumes horaires plus élevés dans les associations. Remarquons que ce sont les deux CS se trouvant aux extrémités de la hiérarchie socioprofessionnelle, à savoir les cadres et les ouvriers, qui apparaissent les plus désavantagées dans les associations. La situation des ouvriers doit cependant être appréhendée avec prudence : beaucoup moins présents en milieu associatif (9\% des postes, contre $21 \%$ dans le reste du privé), certains d'entre eux pourraient être des salariés en insertion dont le contrat n'a pas été identifié comme étant un contrat aidé, ce qui contribuerait à accroître l'écart de rémunération par rapport au reste du secteur privé. En ce qui concerne les cadres, la forte dispersion des salaires annuels, mesurée ici par le coefficient de variation et nettement plus élevée que dans le reste du privé, montre que leur situation n'est pas homogène. Pour les professions intermédiaires et les employés, c'est dans les associations que cette dispersion est au contraire la plus faible. 
TABLEAU 1 - Temps de travail (en heures) et salaires nets annuels (en euros) des postes dans les associations et le secteur privé non associatif

\begin{tabular}{|c|c|c|c|c|c|c|c|}
\hline & & \multicolumn{3}{|c|}{ Associations } & \multicolumn{3}{|c|}{ Secteur privé non associatif } \\
\hline & & Moyenne & Médiane & $C V^{*}$ & Moyenne & Médiane & $C V$ \\
\hline \multirow{2}{*}{$\begin{array}{l}\text { Ensemble des salariés } \\
\text { des services }\end{array}$} & $\begin{array}{l}\text { Temps de travail } \\
\text { Indice }^{* *}\end{array}$ & $\begin{array}{c}1191 \\
94,1\end{array}$ & $\begin{array}{l}1327 \\
90,7\end{array}$ & 0,53 & $\begin{array}{l}1266 \\
100,0\end{array}$ & $\begin{array}{l}1463 \\
100,0\end{array}$ & 0,53 \\
\hline & $\begin{array}{l}\text { Salaire net annuel } \\
\text { Indice }\end{array}$ & $\begin{array}{c}13841 \\
81,4\end{array}$ & $\begin{array}{c}11840 \\
83,7\end{array}$ & 1,84 & $\begin{array}{c}17010 \\
100,0\end{array}$ & $\begin{array}{c}14146 \\
100,0\end{array}$ & 1,52 \\
\hline \multirow{2}{*}{ Cadres } & $\begin{array}{l}\text { Temps de travail } \\
\text { Indice }\end{array}$ & $\begin{array}{l}1205 \\
83,1\end{array}$ & $\begin{array}{l}1418 \\
78,8\end{array}$ & 0,56 & $\begin{array}{l}1450 \\
100,0\end{array}$ & $\begin{array}{l}1799 \\
100,0\end{array}$ & 0,42 \\
\hline & $\begin{array}{l}\text { Salaire net annuel } \\
\text { Indice }\end{array}$ & $\begin{array}{c}22834 \\
63,5\end{array}$ & $\begin{array}{c}21520 \\
70,4\end{array}$ & 2,63 & $\begin{array}{c}35981 \\
100,0\end{array}$ & $\begin{array}{c}30575 \\
100,0\end{array}$ & 1,43 \\
\hline \multirow{2}{*}{$\begin{array}{l}\text { Professions } \\
\text { intermédiaires }\end{array}$} & $\begin{array}{l}\text { Temps de travail } \\
\text { Indice }\end{array}$ & $\begin{array}{l}1222 \\
87,8\end{array}$ & $\begin{array}{c}1429 \\
81,0\end{array}$ & 0,53 & $\begin{array}{l}1392 \\
100,0\end{array}$ & $\begin{array}{l}1764 \\
100,0\end{array}$ & 0,45 \\
\hline & $\begin{array}{l}\text { Salaire net annuel } \\
\text { Indice }\end{array}$ & $\begin{array}{c}15010 \\
78,3\end{array}$ & $\begin{array}{c}14982 \\
79,5\end{array}$ & 0,68 & $\begin{array}{c}19162 \\
100,0\end{array}$ & $\begin{array}{c}18850 \\
100,0\end{array}$ & 1,15 \\
\hline \multirow{2}{*}{ Employés } & $\begin{array}{l}\text { Temps de travail } \\
\text { Indice }\end{array}$ & $\begin{array}{l}1164 \\
102,1\end{array}$ & $\begin{array}{l}1246 \\
106,3\end{array}$ & 0,51 & $\begin{array}{l}1140 \\
100,0\end{array}$ & $\begin{array}{l}1172 \\
100,0\end{array}$ & 0,59 \\
\hline & $\begin{array}{l}\text { Salaire net annuel } \\
\text { Indice }\end{array}$ & $\begin{array}{c}10611 \\
95,7\end{array}$ & $\begin{array}{c}9945 \\
97,3\end{array}$ & 0,68 & $\begin{array}{c}11084 \\
100,0\end{array}$ & $\begin{array}{c}10219 \\
100,0\end{array}$ & 0,79 \\
\hline \multirow{2}{*}{ Ouvriers } & $\begin{array}{l}\text { Temps de travail } \\
\text { Indice }\end{array}$ & $\begin{array}{l}1185 \\
92,9\end{array}$ & $\begin{array}{l}1310 \\
91,7\end{array}$ & 0,53 & $\begin{array}{l}1276 \\
100,0\end{array}$ & $\begin{array}{l}1429 \\
100,0\end{array}$ & 0,55 \\
\hline & $\begin{array}{l}\text { Salaire net annuel } \\
\text { Indice }\end{array}$ & $\begin{array}{c}8719 \\
71,4\end{array}$ & $\begin{array}{c}7546 \\
61,0\end{array}$ & 0,68 & $\begin{array}{c}12210 \\
100,0\end{array}$ & $\begin{array}{c}12369 \\
100,0\end{array}$ & 0,65 \\
\hline
\end{tabular}

* : coefficient de variation.

** : base 100 : temps de travail et salaire dans le secteur privé non associatif.

Lecture : en moyenne, dans l'ensemble des services et toutes CS confondues, l'occupant d'un poste en association perçoit un salaire net annuel de 13841 euros pour un temps de travail de 1191 heures. Par rapport à son homologue du secteur privé non associatif, son salaire est de 18,6\% inférieur (indice de 81,4 pour une base 100 dans le secteur privé non associatif) et son temps de travail est de $5,9 \%$ moins élevé. Le coefficient de variation est un indicateur de la dispersion de la distribution d'une variable. Cette dispersion est d'autant plus forte que le coefficient de variation est élevé.

Champ : postes non annexes du secteur privé, associatif et non associatif, de l'ensemble des services.

Source : Insee-DADS 2010.

Le tableau 2 présente les résultats obtenus par domaine d'activité, pour ceux que nous avons retenus dans l'article. Les salaires annuels des postes associatifs sont supérieurs à ceux du reste du secteur privé dans la santé, le social et le médico-social. S'agissant de ce domaine, l'écart est particulièrement net dans le sous-champ de l'aide à domicile mais il n'est pas négligeable non plus dans les Ehpad. Le constat est tout à fait différent dans le spectacle vivant et les activités de clubs de sports, où les salariés des associations sont moins bien lotis. Dans tous les domaines d'activité, les écarts de salaires se doublent de différences de même signe, sinon de même ampleur, des temps de travail, sauf dans le secteur de la santé où l'on observe peu d'écarts dans les temps de travail.

S'agissant plus précisément des arts du spectacle vivant, en raison du poids de l'intermittence, les postes sont associés à un faible volume horaire moyen et par conséquent offrent des rémunérations plus faibles. Les salariés des associations pâtissent davantage de cette situation. C'est toutefois dans les activités des clubs de 
TABLEAU 2 - Temps de travail (en heures) et salaires nets annuels (en euros) des postes dans les associations et le secteur privé non associatif et dans plusieurs domaines d'activité

\begin{tabular}{|c|c|c|c|c|c|c|c|}
\hline & & \multicolumn{3}{|c|}{ Associations } & \multicolumn{3}{|c|}{ Secteur privé non associatif } \\
\hline & & Moyenne & Médiane & $C V^{*}$ & Moyenne & Médiane & $C V$ \\
\hline \multirow{4}{*}{ Social et médico-social } & Temps de travail & 1225 & 1365 & \multirow[t]{2}{*}{0,48} & 1058 & 1005 & \multirow[t]{2}{*}{0,60} \\
\hline & Indice $^{* * *}$ & 115,8 & 135,8 & & 100,0 & 100,0 & \\
\hline & Salaire net annuel & 12235 & 11018 & \multirow[t]{2}{*}{0,69} & 10722 & 9290 & \multirow[t]{2}{*}{0,80} \\
\hline & Indice & 114,1 & 118,6 & & 100,0 & 100,0 & \\
\hline \multirow{4}{*}{ Ehpad } & Temps de travail & 1178 & 1347 & \multirow[t]{2}{*}{0,52} & 1098 & 1101 & \multirow[t]{2}{*}{0,58} \\
\hline & Indice & 107,3 & 122,3 & & 100,0 & 100,0 & \\
\hline & Salaire net annuel & 12110 & 12255 & \multirow[t]{2}{*}{0,65} & 11200 & 10865 & \multirow[t]{2}{*}{0,69} \\
\hline & Indice & 108,1 & 112,8 & & 100,0 & 100,0 & \\
\hline \multirow{4}{*}{ Aide à domicile } & Temps de travail & 1059 & 1098 & \multirow[t]{2}{*}{0,50} & 818 & 704 & \multirow[t]{2}{*}{0,66} \\
\hline & Indice & 129,5 & 156,0 & & 100,0 & 100,0 & \\
\hline & Salaire net annuel & 8750 & 8384 & \multirow[t]{2}{*}{0,61} & 6774 & 5466 & \multirow[t]{2}{*}{0,82} \\
\hline & Indice & 129,2 & 153,4 & & 100,0 & 100,0 & \\
\hline \multirow{4}{*}{ Santé } & Temps de travail & 1312 & 1524 & \multirow[t]{2}{*}{0,46} & 1297 & 1509 & \multirow[t]{2}{*}{0,48} \\
\hline & Indice & 101,2 & 101,0 & & 100,0 & 100,0 & \\
\hline & Salaire net annuel & 18841 & 16424 & \multirow[t]{2}{*}{0,83} & 15409 & 14816 & \multirow[t]{2}{*}{0,74} \\
\hline & Indice & 122,3 & 110,99 & & 100,0 & 100,0 & \\
\hline \multirow{4}{*}{$\begin{array}{l}\text { Arts du spectacle } \\
\text { vivant }\end{array}$} & Temps de travail & 651 & 460 & \multirow[t]{2}{*}{0,82} & 718 & 480 & \multirow[t]{2}{*}{0,84} \\
\hline & Indice & 90,7 & 95,8 & & 100,0 & 100,0 & \\
\hline & Salaire net annuel & 9352 & 5899 & \multirow[t]{2}{*}{8,03} & 11763 & 6861 & \multirow[t]{2}{*}{1,60} \\
\hline & Indice & 79,5 & 86,0 & & 100,0 & 100,0 & \\
\hline \multirow{4}{*}{ Sports et loisirs } & Temps de travail & 869 & 652 & 0,76 & 1060 & 912 & 0,67 \\
\hline & Indice & 82,0 & 71,5 & & 100,0 & 100,0 & \\
\hline & Salaire net annuel & 9916 & 7021 & 1,21 & 19335 & 8741 & 4,78 \\
\hline & Indice & 51,3 & 80,3 & & 100,0 & 100,0 & \\
\hline & Temps de travail & 866 & 648 & 0,76 & 1524 & 1820 & 0,52 \\
\hline Activités de clubs & Indice & 56,8 & 35,6 & & 100,0 & 100,0 & \\
\hline de sports & Salaire net annuel & 9979 & 7171 & 1,18 & 77340 & 21665 & 3,02 \\
\hline & Indice & 12,9 & 33,1 & & 100,0 & 100,0 & \\
\hline & Temps de travail & 879 & 717 & 0,73 & 817 & 580 & 0,76 \\
\hline Autres activités & Indice & 107,6 & 123,6 & & 100,0 & 100,0 & \\
\hline récréatives et de loisirs & Salaire net annuel & 8389 & 6399 & 0,87 & 7997 & 5135 & 0,99 \\
\hline & Indice & 104,9 & 124,6 & & 100,0 & 100,0 & \\
\hline
\end{tabular}

* : coefficient de variation.

**: base 100 : temps de travail et salaire dans le secteur privé non associatif.

Des tests paramétriques (respectivement non paramétriques) conduisent à rejeter, au seuil de 0,001 , l'hypothèse d'égalité des moyennes (respectivement des médianes) aussi bien pour les salaires que pour les volumes horaires dans chacun des secteurs (associations et privé non associatif).

Lecture : l'occupant d'un poste dans le domaine social et médico-social associatif perçoit en moyenne un salaire net annuel de 12235 euros, soit de 14,1\% supérieur à celui de son homologue dans le secteur privé non associatif dont le salaire est de 10722 euros. Champ : postes non annexes du secteur privé associatif et non associatif.

Source : Insee-DADS 2010.

sports que l'écart des salaires annuels est le plus élevé entre associations et reste du privé. Il est ici considérable puisque le salaire médian dans les premières ne représente que $33 \%$ de celui en vigueur dans le second. Il s'explique par les fortes rémunérations des sportifs professionnels dans le secteur privé non associatif, qui contribuent à tirer vers le haut les salaires. Au cours des dernières décennies, nombre de clubs sportifs 
professionnels (dans le football et le rugby notamment) ont changé de statut juridique et abandonné leur ancien statut associatif pour adopter celui de société anonyme, ce qui a accru la différence de situation économique (et donc de salaire en leur sein) des clubs selon qu'ils sont, ou non, restés des associations. Enfin, dans les autres activités récréatives et de loisirs (par exemple, dans les parcs de loisirs, les stations de ski, etc.), les temps de travail sont là encore fort courts, quoique supérieurs à ceux du spectacle vivant, mais ils sont encore plus courts dans le reste du secteur privé où le salaire annuel est également plus faible que dans les associations. Dans ce sous-domaine, à la différence du sport et du spectacle vivant, notre hypothèse de rémunérations associatives plus faibles ne paraît donc pas confirmée.

Ces résultats, qui permettent de documenter la diversité des situations selon la nature de l'activité, doivent toutefois être interprétés avec prudence puisqu'ils ne sont pas corrigés de la composition des effectifs salariés, qui peut différer entre les deux secteurs dans chacun des domaines. Lorsque le même type d'examen est effectué par $\mathrm{CS}$, les constats (non reproduits ici) montrent que c'est dans le spectacle vivant ainsi que dans les sports et les loisirs que les cadres associatifs subissent un fort différentiel négatif de salaire annuel par rapport à leurs semblables du reste du privé. Par exemple, dans les autres activités récréatives et de loisirs, le salaire médian d'un poste de cadre en milieu associatif est de $45 \%$ moins élevé que celui de son homologue dans le reste du privé, et son temps de travail annuel médian y est de $50 \%$ plus faible. Pour les professions intermédiaires, les constats convergent en partie avec ceux relatifs aux cadres : dans les associations du spectacle vivant, leurs rémunérations sont également plus faibles que dans le reste du privé ; et l'écart est encore plus conséquent dans les activités de clubs de sports, en raison notamment de la présence des sportifs professionnels, qui sont classés dans cette $\mathrm{CS}^{15}$. En revanche, et contrairement aux cadres, dans les autres activités récréatives et de loisirs, les salaires des professions intermédiaires associatives ne diffèrent guère de ceux observés dans le reste du privé. Pour les employés, la comparaison n'est défavorable aux associations que dans le domaine des activités de clubs de sports, tandis que pour les ouvriers, elle l'est également dans le spectacle vivant ainsi que dans le domaine social et médico-social considéré dans son ensemble.

Les constats qui précèdent concernent les postes de travail. Or un même salarié peut en occuper plusieurs dans des établissements différents. Dans les associations, $59 \%$ des postes sont occupés à titre exclusif, contre $64 \%$ dans le reste du secteur privé. Sans surprise, on constate une relation positive entre les temps de travail moyens des postes (tableau 2) et la part des postes occupés à titre exclusif (tableau 3). Dans le spectacle vivant, le sport et les loisirs, cette proportion est plus faible dans les associations que dans le reste du privé. On observe l'inverse dans le social et le médico-social dans leur ensemble, et dans les Ehpad en particulier. C'est aussi le cas dans les secteurs de l'aide à domicile et de la santé mais les écarts y sont modestes.

15. Il est impossible de les soustraire de notre investigation dans la mesure où ils font partie du même poste de niveau 3 de la nomenclature socioprofessionnelle que les moniteurs et éducateurs sportifs. 
TABLEAU 3 - Part des postes occupés à titre exclusif

En \%

\begin{tabular}{lcc}
\hline & Associations & Secteur privé non associatif \\
\hline Ensemble des services & 59,1 & 64,0 \\
\hline Social et médico-social & 61,9 & 54,6 \\
$\quad$ Ehpad & 64,9 & 57,2 \\
$\quad$ Aide à domicile & 45,6 & 43,4 \\
\hline Santé & 66,1 & 65,1 \\
\hline Arts du spectacle vivant & 27,6 & 30,8 \\
\hline Sports et loisirs & 40,8 & 59,7 \\
$\quad$ Activités de clubs de sports & 39,7 & 62,9 \\
$\quad$ Activités récréatives et de loisirs & 47,2 & 50,3 \\
\hline
\end{tabular}

Champ : postes non annexes du secteur privé, associatif et non associatif.

Source : Insee-DADS 2010.

Lorsque le salarié n'occupe qu'un seul poste, il n'est pas surprenant de remarquer que ce dernier se caractérise par un volume horaire et une rémunération annuelle supérieurs à la moyenne des postes. Toutefois, s'agissant de la nature des différences observées ${ }^{16}$, même si celles-ci sont alors généralement réduites par rapport à celles du tableau 2, les comparaisons entre les deux secteurs ne produisent pas de résultats substantiellement dissemblables de ceux obtenus précédemment.

En résumé, la comparaison entre les salaires annuels associatifs et ceux du reste du privé montre que les écarts observés, tantôt en faveur des premiers (dans la santé, le social et le médico-social et même dans les autres activités récréatives et de loisirs), tantôt à leur détriment (dans le spectacle vivant et le sport), ne sont pas sans lien avec la différence des temps de travail. Mais cette dernière n'épuise pas l'explication des différentiels salariaux, qui relèvent également de rémunérations horaires inégales, comme nous allons le voir.

\section{Analyse descriptive et toutes choses égales par ailleurs des salaires horaires}

Le tableau 4 présente les salaires horaires nets dans le secteur associatif et dans le reste du secteur privé. Pour l'ensemble des services, le salaire moyen du premier est inférieur à celui du second mais le salaire médian y est légèrement plus élevé et la dispersion salariale plus faible. Les disparités sont importantes selon les domaines d'activité. Une fois encore, la comparaison est nettement défavorable pour les salariés associatifs du spectacle vivant et pour ceux des activités de clubs de sports, tandis que dans la santé, mais aussi dans les autres activités récréatives et de loisirs, ce sont au contraire les associations qui offrent les salaires horaires les plus élevés. Dans le social et le médico-social, les écarts sont plutôt minimes et peuvent paraître soit favorables soit défavorables selon l'indicateur retenu (moyenne ou médiane).

16. Ces comparaisons ne sont pas reproduites ici mais disponibles auprès des auteurs. 
TABLEAU 4 - Salaires nets horaires (en euros) dans les associations et le secteur privé non associatif

\begin{tabular}{lrrrrrrr}
\hline & \multicolumn{3}{c}{ Associations } & & \multicolumn{3}{c}{ Secteur privé non associatif } \\
\cline { 2 - 3 } & Moyenne & Médiane & $C V^{*}$ & & Moyenne & Médiane & $C V$ \\
\hline Ensemble des services & 11,57 & 9,92 & 1,41 & 12,72 & 9,83 & 3,31 \\
\hline Social et médico-social & 10,02 & 9,13 & 0,45 & & 10,09 & 9,02 & 0,43 \\
$\quad$ Ehpad & 10,47 & 9,57 & 0,38 & & 10,44 & 9,37 & 0,39 \\
$\quad$ Aide à domicile & 8,27 & 7,74 & 0,27 & 8,15 & 7,62 & 0,27 \\
\hline Santé & 14,39 & 11,63 & 0,66 & 12,00 & 10,31 & 0,54 \\
\hline Arts du spectacle vivant & 14,51 & 12,82 & 2,10 & 16,72 & 14,29 & 0,65 \\
\hline Sports et loisirs & 12,04 & 10,11 & 1,16 & & 13,94 & 8,89 & 3,55 \\
$\quad$ Activités de clubs de sports & 9,62 & 8,71 & 0,40 & & 9,44 & 8,25 & 3,65 \\
$\quad$ Autres activités récréatives et de loisirs & 12,46 & 10,52 & 1,23 & & 39,21 & 15,81 & 2,72 \\
\hline
\end{tabular}

* : coefficient de variation.

Note : des tests paramétriques (respectivement non paramétriques) conduisent à rejeter, au seuil de $0,001,1$ l'hypothèse d'égalité des moyennes (respectivement des médianes) des salaires horaires dans les deux secteurs sauf dans les autres activités récréatives et de loisirs, où cette hypothèse est rejetée au seuil de 0,003 et dans les Ehpad, où l'hypothèse d'égalité des moyennes n'est pas rejetée aux seuils conventionnels de significativité.

Lecture : le salaire net horaire moyen est de 11,57 euros en association contre 12,72 euros dans le secteur privé non associatif, mais le coefficient de variation est inférieur dans les premières (1,41 contre 3,31 dans le second). L'échelle des salaires horaires est donc plus dispersée dans le secteur privé non associatif.

Champ : postes non annexes du secteur privé, associatif et non associatif.

Source : Insee-DADS 2010.

Les comparaisons ci-dessus ne tiennent pas compte des différences de caractéristiques des postes, des salariés et des entreprises. Ainsi, dans le domaine de la santé, si le salaire horaire dans les associations est plus élevé que dans le reste du privé, c'est notamment parce que les cadres y sont très présents (près de $16 \%$ des postes, contre $6 \%$ dans le reste du privé), en raison des activités de recherche qu'ils y exercent et qui nécessitent un haut niveau de qualification (BISAUlt, 2012). Pour corriger ces effets de composition, nous avons réalisé une investigation économétrique dans un premier temps au niveau agrégé, c'est-à-dire au niveau de l'ensemble des services. Dans le modèle estimé, la variable dépendante est le logarithme du salaire horaire net. La variable explicative d'intérêt est une variable dichotomique prenant la valeur 1 si l'employeur est de type associatif et 0 s'il appartient au reste du privé. Les variables de contrôle sont le sexe du salarié occupant le poste, son âge (sous forme quadratique), le type de contrat (contrat à durée indéterminée [CDI] ou contrat à durée déterminée [CDD]), le type d'emploi (temps plein ou temps partiel), la taille de l'entreprise, la région d'implantation de l'établissement et le domaine d'activité. Pour ce dernier, nous avons effectué une partition des services en quinze domaines. Comme nous l'avons déjà noté, les $D A D S$ ne permettent de connaître ni le niveau de diplôme ni l'ancienneté du salarié dans l'entreprise. En lieu et place de ces variables manquantes, qui nous interdisent de construire une véritable équation de salaire mincerienne, nous utilisons la CS (à un chiffre) du poste occupé. 
L'estimation se fait successivement sans puis avec le contrôle des domaines d'activité. Seuls sont présentés ici les coefficients associés à la variable d'intérêt, c'est-à-dire ceux relatifs au statut de l'employeur ${ }^{17}$. L'introduction des domaines d'activité a ainsi pour conséquence une diminution substantielle de l'écart négatif estimé de rémunération entre les associations et le reste du secteur privé qui passe de près de $10 \%$ à environ $4 \%(\text { tableau } 5)^{18}$. Un tel résultat fait écho à ceux des études menées aux États-Unis et évoquées dans notre première partie. Il confirme bien la concentration de l'emploi associatif dans des domaines où la rémunération est plutôt faible. Par conséquent, pour que la comparaison entre organismes de statuts juridiques différents ait un sens, il est nécessaire de la mener à un niveau désagrégé. L'exercice est réalisé dans un second temps en estimant une équation de salaire dans chacun des domaines retenus pour cette étude.

\section{TABLEAU 5 - L'effet du statut associatif sur les salaires horaires}

\begin{tabular}{|c|c|c|c|c|c|}
\hline Domaine d'activité & $\begin{array}{l}\text { Coefficient } \\
\text { de la variable } \\
\text { relative aux } \\
\text { associations }\end{array}$ & Écart-type & $\begin{array}{l}\text { Nombre } \\
\text { total } \\
\text { de postes }\end{array}$ & $\begin{array}{c}\text { Nombre } \\
\text { de postes } \\
\text { en association }\end{array}$ & R2 ajusté \\
\hline \multicolumn{6}{|c|}{ Estimation au niveau agrégé (ensemble des services) } \\
\hline $\begin{array}{l}\text { Sans les domaines d'activité parmi } \\
\text { les variables de contrôle }\end{array}$ & $-0,1026^{* * *}$ & 0,0002 & 13437212 & 1900763 & 0,580 \\
\hline $\begin{array}{l}\text { Avec les domaines d'activité comme } \\
\text { variables de contrôle }\end{array}$ & $-0,0443^{* * *}$ & 0,0003 & 13437212 & 1900763 & 0,592 \\
\hline \multicolumn{6}{|l|}{ Estimation par domaine d'activité } \\
\hline Social et médico-social & $-0,0179^{* * *}$ & 0,0007 & 1083889 & 856478 & 0,536 \\
\hline Ehpad & $0,0083^{* * *}$ & 0,0011 & 185869 & 85321 & 0,461 \\
\hline Aide à domicile & $0,0059^{* * *}$ & 0,0010 & 225020 & 177844 & 0,264 \\
\hline Santé & $-0,0085^{* * *}$ & 0,0009 & 607220 & 146053 & 0,555 \\
\hline Arts du spectacle vivant & $-0,0862^{* * *}$ & 0,0035 & 71119 & 45738 & 0,297 \\
\hline Sport et loisirs & $-0,1929^{* * *}$ & 0,0028 & 149812 & 79995 & 0,342 \\
\hline Activités de clubs de sports & $-0,5191^{*}$ & 0,0065 & 68188 & 61363 & 0,332 \\
\hline Autres activités récréatives et de loisirs & $-0,0306^{* * *}$ & 0,0038 & 24523 & 10008 & 0,316 \\
\hline \multicolumn{6}{|l|}{$* * *$ : significatif au seuil de 0,0001 . } \\
\hline \multicolumn{6}{|c|}{$\begin{array}{l}\text { Lecture : pour l'équation de salaire dans l'ensemble des services, le coefficient associé au fait d'être en association est négatif, ce qui } \\
\text { signifie que les salaires y sont inférieurs à ceux du secteur privé non associatif. L'écart diminue toutefois quand les domaines d'activité } \\
\text { sont introduits dans le modèle (le coefficient passe de-0,1026 à -0,0443). Les écarts de salaires en pourcentage peuvent être calculés } \\
\text { selon la formule de la note } 18 \text {. }\end{array}$} \\
\hline
\end{tabular}

17. Les résultats complets sont disponibles auprès des auteurs. Les coefficients associés aux autres variables explicatives ont le signe attendu : le salaire horaire augmente à taux décroissant avec l'âge du salarié, il est plus faible chez les femmes, il augmente avec l'élévation du poste dans la hiérarchie des CS, il est supérieur pour les temps pleins et légèrement plus faible pour les CDD. Il est positivement corrélé à l'augmentation de la taille de l'entreprise et il est plus élevé en Île-de-France.

18. Dans un modèle semi-logarithmique comme celui qui est présentement estimé, la variation en pourcentage du salaire horaire induite par un changement de la valeur de la variable dichotomique d'intérêt de 0 à 1 (ici le passage d'un employeur du secteur privé à un employeur associatif) est calculée selon la formule : $100 \times\left(\mathrm{e}^{\beta}-1\right)$ où $\beta$ est le coefficient estimé de la variable en question. 
C'est de nouveau dans les activités de clubs de sports et dans le spectacle vivant que les salariés associatifs subissent le plus fort déficit de rémunération horaire, comparativement à ceux du reste du privé. Dans les activités de clubs de sports, la présence des sportifs professionnels dans le reste du privé explique encore une fois une part substantielle mais impossible à discerner du fort différentiel constaté. Dans le spectacle vivant, les salaires horaires associatifs sont inférieurs d'un peu plus de $8 \%$ à ceux du reste du secteur privé. Mais cette fois-ci, contrairement à ce que suggéraient les statistiques descriptives du tableau 4, les salariés associatifs des autres activités récréatives et de loisirs apparaissent également pénalisés par rapport aux salariés du reste du privé, l'écart étant toutefois plus faible (3\%) que dans les deux champs précédents. Dans la santé, l'écart observé dans le tableau 4 en faveur des salariés associatifs disparaît et se transforme en différence négative, d'une importance toutefois mineure (inférieure à $1 \%$ ) .

Dans le social et le médico-social pris dans leur ensemble, la différence de salaire horaire est au détriment des salariés des associations même si elle reste là encore d'une ampleur limitée (environ 1,5\%). En revanche dans les Ehpad comme dans l'aide à domicile, on observe une situation de quasi-parité entre les salariés associatifs et leurs homologues du reste du privé, un très léger avantage revenant même aux premiers.

Les résultats obtenus par l'étude empirique en termes de rémunérations annuelles et de rémunérations horaires sont-ils cohérents avec les hypothèses auxquelles nous conduisait notre deuxième partie ? La réponse est, dans une large mesure, affirmative mais nécessite d'être nuancée. Certes, dans des domaines comme le sport et le spectacle vivant, les rémunérations associatives, tant annuelles qu'horaires, sont inférieures, et parfois nettement, à celles du reste du secteur privé. C'est donc bien dans ces domaines que la présomption d'un don de travail semble la plus plausible. En revanche dans les secteurs de la santé, des Ehpad ainsi que de l'aide à domicile, les salaires horaires dans les associations sont d'un niveau à peu près équivalent à ceux du reste du secteur privé et les rémunérations annuelles y sont plus élevées du fait de temps de travail plus importants. La nuance à apporter concerne le champ des autres activités récréatives et de loisirs. Nous présumions que leurs salariés percevraient des salaires inférieurs à ceux du reste du secteur privé. L'hypothèse est vérifiée pour les salaires horaires. En revanche les rémunérations annuelles sont supérieures dans les associations, en raison de temps de travail plus importants. Ainsi, si la précarité de l'emploi est forte dans les deux secteurs, elle l'est davantage encore dans le reste du privé où le dialogue social de branche s'est concrétisé plus tardivement, puisque la convention collective le concernant a été signée plusieurs années après celle de l'animation, en janvier $1994^{19}$.

19. Il s'agit de la convention collective nationale des espaces de loisirs, d'attractions et culturels, qui s'applique aux entreprises de droit privé à but lucratif. Un examen plus détaillé révèle que cet écart de salaire annuel est essentiellement le fait des postes occupés par les employés dont le temps annuel médian est deux fois supérieur dans les associations. L'explication de cette situation demande approfondissement. 
S'agissant de la comparaison entre les salaires dans les associations et ceux dans le reste du secteur privé, les hypothèses émises dans la littérature économique divergent ; cependant, celle relative au don de travail des salariés associatifs a largement été reprise. Si les approches au fondement de ces hypothèses ne manquent pas d'intérêt, elles relèguent néanmoins à l'arrière-plan les dimensions institutionnelles et historiques de la relation salariale. Or leur prise en compte nous semble nécessaire pour conduire un tel exercice comparatif car elle permet de mieux comprendre l'hétérogénéité des résultats qui en découlent lorsqu'il est conduit à un niveau désagrégé de la nomenclature des activités. Nous avons mis plus particulièrement l'accent sur le rôle de deux facteurs qui, dans une certaine mesure, sont interdépendants. Le premier concerne l'intensité du recours au bénévolat. Le second renvoie à l'histoire des processus de professionnalisation des associations, marquée par des décalages chronologiques en fonction des domaines d'activité, décalages qui ont laissé leur empreinte sur les relations professionnelles, et notamment sur le rôle joué par les accords collectifs dans la relation salariale. Notre hypothèse était que les salaires dans les associations sont d'autant plus susceptibles d'être inférieurs à ceux du reste du privé que le recours au bénévolat y est important et que la mise en œuvre du dialogue social de branche a été tardive. Notre investigation empirique présente l'originalité de prendre en compte à la fois le salaire horaire et la rémunération annuelle. Les résultats confirment notre hypothèse en montrant que c'est dans les domaines du sport et du spectacle vivant, qui mobilisent de nombreux bénévoles et dont la professionnalisation est récente, que l'écart des rémunérations au détriment des salariés associatifs est le plus net, si bien que ces domaines sont des champs de prédilection pour l'hypothèse de don de travail avancée dans la littérature économique. Dans le secteur de la santé, du social et du médico-social comme les Ehpad ou l'aide à domicile, les salaires horaires estimés toutes choses égales par ailleurs ne diffèrent guère entre les deux secteurs mais, plus particulièrement dans le social et le médico-social, les rémunérations annuelles moyennes sont plus importantes dans les associations du fait de temps de travail supérieurs. La situation dans les loisirs, domaine également fortement utilisateur de bénévolat, se révèle quelque peu atypique car si le salaire horaire estimé par le traitement économétrique est inférieur dans les associations, les rémunérations annuelles moyennes y sont plus élevées, ce qui s'explique en partie par des temps de travail plus importants que dans le reste du privé.

Notre étude n'est pas sans limites. En particulier, elle ne tient pas compte du temps de travail non rémunéré susceptible d'être réalisé par les salariés : en effet, il pourrait affecter nos résultats s'il était d'une ampleur différente dans les deux secteurs. Une telle information est bien sûr absente de la source que nous avons utilisée, les $D A D S$, qui ne renseignent que le temps de travail rémunéré. À notre connaissance, aucune autre source française ne peut être mobilisée sur ce point.

Notre article appelle aussi des prolongements dans au moins deux directions. La première consisterait à étendre la comparaison entre associations et reste du privé à d'autres domaines d'activité que ceux retenus ici : si ces derniers sont importants au 
sein du monde associatif, notamment eu égard au volume d'emplois qu'ils y représentent, ils n'en épuisent pour autant pas la diversité. Nous pensons par exemple à l'enseignement, à la formation continue et au tourisme. La seconde direction consisterait, quant à elle, en une étude moins intuitive et plus empiriquement documentée de l'effet de la présence des ressources bénévoles sur les salaires à une échelle microéconomique (celui de l'organisation) et non à celle méso-économique (le domaine d'activité) qui seule a été ici envisagée. Les recherches sur le sujet sont extrêmement rares. Le travail d'Astrid HAIDER et Ulrike SCHNEIDER (2010) fait exception, mais ses enseignements sont d'une portée limitée. Il montre que la présence de bénévoles dans l'organisation s'accompagne effectivement de salaires plus faibles mais que le nombre de bénévoles semble sans effet sur la rémunération du personnel. Les auteurs ne disposent toutefois pas de précisions sur le volume de ce travail bénévole, alors qu'il constitue pourtant une variable essentielle pour une telle analyse. Il y a donc là une voie de recherche prometteuse qui suppose l'existence d'une base de données offrant une information conjointe sur l'emploi salarié et le bénévolat. L'enquête Associations 2014 que l'Insee a récemment réalisée ouvre des perspectives en ce sens (REYNAERT, D’ISANTO, 2016).

\section{BIBLIOGRAPHIE}

Bazzoli L., KiRAT T., Villeval M.-C. (1994), « Contrat et institutions dans la relation salariale : pour un renouveau institutionnaliste », Travail et Emploi, ${ }^{\circ}$ 58, pp. 94-110.

Bisault L. (2012), «L'échelle des salaires est plus resserrée dans le secteur de l'économie sociale », Insee première, $\mathrm{n}^{\circ} 1390$.

BouRdoncle R. (2000), « Professionnalisation, formes et dispositifs », Recherche et formation, $\mathrm{n}^{\mathrm{o}} 35$, pp. 117-132.

BUREAU M.-C., SCHMidT N. (2004), « Les emplois-jeunes dans la culture, une politique sectorielle ? », Connaissance de l'emploi, $\mathrm{n}^{\circ} 7$, Noisy-le-Grand, Centre d'études de l'emploi.

Chantelat P. (2001) (textes réunis et présentés par), La Professionnalisation des organisations sportives, Paris, L'Harmattan.

Снімот С., Sснотте́ M. (2006), « Travailler dans une organisation sportive. Entre engagement passionné et investissement professionnel », Regards sociologiques, $\mathrm{n}^{\circ}$ 32, pp. 97-107.

CONSEIL D'ÉTAT (2000), « Les associations et la loi de 1901, cent ans après », in Rapport Public 2000, pp. 237-422.

Corsani A., LazZarato M. (2008), Intermittents et précaires, Paris, Éditions Amsterdam.

Deroin V. (2014), «Emploi, bénévolat et financement des associations culturelles », Culture chiffres, $\mathrm{n}^{\circ}$ 2014-1, Ministère de la Culture et de la Communication, Département des études, de la prospective et des statistiques.

DuRAND J. (2014), «Que sont devenues les personnes sorties de contrats aidés en 2012 ? », Dares Analyses, $\mathrm{n}^{\circ} 071$. 
FAlcoz M., Walter E. (2007), « Travailler dans un monde de bénévoles. Contraintes et limites de la professionnalisation dans les clubs sportifs », Recma-Revue internationale de l'économie sociale, $\mathrm{n}^{\mathrm{o}} 306, \mathrm{pp} .78-91$.

Fleuriel S. (2013), « Ce que dialoguer veut dire. L'émergence du "dialogue social” dans le sport français (1990-2006) », Genèses, n 92, pp. 127-146.

Gouyon M., Patureau F. (2014), «Vingt ans d'évolution de l'emploi dans les professions culturelles (1991-2011) », Culture chiffres, $\mathrm{n}^{\circ}$ 2014-6, Ministère de la Culture et de la Communication, Département des études, de la prospective et des statistiques.

Haider A., Schneider U. (2010), “The Influence of Volunteers, Donations and Public Subsidies on the Wage Level of Nonprofit Workers: Evidence from Austrian Matched Data", Annals of Public and Cooperative Economics, vol. 81, $\mathrm{n}^{\mathrm{o}}$ 1, pp. 1-20.

Handy F., Katz E. (1998), “The Wage Differential between Nonprofit Institutions and Corporations: Getting More by Paying Less?", Journal of Comparative Economics, vol. 26, $\mathrm{n}^{\mathrm{o}} 2$, pp. 246-261.

Hansmann H. B. (1980), “The Role of Nonprofit Enterprise”, The Yale Law Journal, vol. 89, $\mathrm{n}^{\mathrm{o}} 5$, pp. 835-901.

HéLy M. (2008), « À travail égal salaire inégal : ce que travailler dans le secteur associatif veut dire », Sociétés contemporaines, $\mathrm{n}^{\circ}$ 69, pp. 125-147.

Hély M. (2009), Les Métamorphoses du monde associatif, Paris, Presses universitaires de France.

Hély M., RÉTIF S., Simonet M. (2015), « Figures de l'employeur et formes du “dialogue social” dans les entreprises de l'ESS », Mouvements, $\mathrm{n}^{\circ} 81$, pp. 116-125.

Ion J. (2005), « Brève chronique des rapports entre travail social et bénévolat », Pensée plurielle, $\mathrm{n}^{\circ} 10$, pp. 149-157.

LE RENDU-LizÉE C. (2005), « Quelles formes d'implication dans les lieux de musiques actuelles et amplifiées ?», Volume!, vol. 4, n 2, pp. 25-40.

Lebon F. (2009), Les Animateurs socioculturels, Paris, La Découverte.

LEETE L. (2001), "Wither the Nonprofit Wage Differential? Estimates from the 1990 Census", Journal of Labor Economics, vol. 19, n 1, pp. 136-170.

LeETE L. (2006), "Work in the Nonprofit Sector”, in Powell W. W., Steinberg R. (eds), The Nonprofit Sector: A Research Handbook, New Haven (Connecticut), London, Yale University Press, pp. 159-179.

Menger P.-M. (2010), «Les professions culturelles : un système incomplet de relations sociales », in Poirrier P., Politiques et pratiques de la culture, Paris, La Documentation française, pp. 243-253.

Meurs D., PonthieuX S. (2006), « L'écart des salaires entre les hommes et les femmes peut-il encore baisser ? », Économie et statistique, n ${ }^{\circ} 398-399$, pp. 99-129.

MiAs C. (1998), L'Implication professionnelle dans le travail social, Paris, L'Harmattan. 
NARCY M. (2009), «Les salariés du secteur associatif sont-ils davantage intrinsèquement motivés que ceux du secteur privé ? », Économie et prévision, n ${ }^{\circ}$ 188, pp. 81-99.

Preston A. E. (1988), “The Effects of Property Rights on Labor Costs of Nonprofit Firms: An Application to the Day Care Industry", The Journal of Industrial Economics, vol. 36, $\mathrm{n}^{\circ} 3$, pp. 337-350.

Preston A. E. (1989), “The Nonprofit Worker in a For-Profit World”, Journal of Labor Economics, vol. 7, $\mathrm{n}^{\circ}$ 4, pp. 438-463.

Preston A. E., Sacks D. W. (2010), "Nonprofit Wages: Theory and Evidence", in Seaman B. A., Young D. R. (eds), Handbook of Research on Nonprofit Economics and Management, Cheltenham, Edward Elgar, pp. 106-119.

Proust S. (2010), « Syndicalisme et délitement du salariat artistique : la CGT et les groupes mobilisés autour du régime de l'intermittence », Sociologie du travail, vol. 52, n 3, pp. 374-388.

Proust S. (2012), «L'impossible transfert des règles de la société salariale dans les champs artistiques : l'exemple d'un dispositif de qualification dans le spectacle vivant », Formation emploi, $\mathrm{n}^{\mathrm{o}} 119$, pp. 65-81.

Ravon B., Ion J. (2012), Les Travailleurs sociaux, 8 édition, Paris, La Découverte.

REYNAERT L., D’IsANTO A. (2016), « Neuf associations sur dix fonctionnent sans salarié », Insee première, $\mathrm{n}^{\mathrm{o}} 1586$.

Rosen S. (1986), "The Theory of Equalizing Differences”, in Ashenfelter O., Layards R. (eds), Handbook of Labor Economics, vol. 1, Amsterdam, Oxford, North Holland, pp. 641-692.

RuHM C. J., Borkoski C. (2003), "Compensation in the Nonprofit Sector", The Journal of Human Resources, vol. 38, n 4, pp. 992-1021.

Simonet M. (2006), « Le monde associatif : entre travail et engagement », in Alter N. (dir.), Sociologie du monde du travail, Paris, Presses universitaires de France, pp. 191-207.

TCHERnonog V. (2013), Le Paysage associatif français, mesures et évolutions : économie, sociologie, 2 édition, Paris, Dalloz; Lyon, Juris éditions.

Urrutiaguer D. (2009), Économie et droit du spectacle vivant en France, Paris, Presses Sorbonne nouvelle.

Weisbrod B. A. (1983), "Nonprofit and Proprietary Sector Behavior: Wage Differential among Lawyers", Journal of Labor Economics, vol. 1, n 3, pp. 246-263. 